\title{
Development of the Children's Abilities in School. A comparative study between the efficiency of the Robotics vs Applied Mathematics in Movement Transmission
}

\author{
Ana-Maria Popa•
}

\begin{abstract}
A qualitative and comparative analysis is performed regarding the possible consequences of the introduction in the school curriculum of 10-12 years old children, of Robotics and Applied Mathematics in Movement Transmission (AMMT) courses, respectively. The research has been done at the Gymnazial School Number 195, from Bucharest, during two scholar years (2017-2019). Each class had 36 students and the two hours Robotics / AMMT courses had a weekly frequency. Direct observation, interviews of the children and parents, together with the class and individual journals, where children noted their opinions during the two years, are analyzed and interpreted. The results show better scholar performances of the children following Robotics courses, in respect to the ones following AMMT ones and a greater attraction of the children in Robotics. Further, a quantitative evaluation regarding the improvement of mathematical performances of children is presented, given a very similar level of the two classes at the beginning of the research in respect to their average Maths scores. The analysis involved several statistical parameters, like the average scores obtained at Math tests, their variances, the evolution of the children with the lowest performances and the number of the maximum scores in each class. All of them suggest the Robotics course efficiency in increasing mathematical performances level of young children is superior to the one of the AMMT course, recommending the introduction of such courses in the school curriculum.
\end{abstract}

Keywords: math; networking; performance; robotics; students

\section{Introduction}

Continuous development of various commercial Robotic kits dramatically changed the classic model of the mechanical toys or puppets. Consequently, the study of Robotics is now extended at a large scale in schools of various degrees in the USA and all over the world (Rogers et al., 2010). Educative robotic kits have been designed by both commercial companies like LEGO and research labs in universities (Martin et al., 2000; Rogers \& Portsmore, 2004; Rusk et al., 2008). Waterhouse (2005) outlined that most of the Robotic courses in schools are related to the concept of education focused on the individual child. A major characteristic of such an approach is the development of certain

\footnotetext{
- Ana-Maria Popa - PhD. candidate, Educational Sciences, Bucharest University, Romania, ana.maria.popa@drd.unibuc.ro
} 
abilities, not only of some knowledge specific to a curriculum. Robotic courses may become important sources of development of both manual and abstract abilities for the children. So, they may be engineers by exploring the tools, electrical engines and sensors, but also they may tell us stories by creating some artefacts responding to the ambient (Bers, 2008). Robotics may help a better understanding of the basic concepts of Math, like the number, dimension and shape. Also, it provides a proper methodology for scientific research and the best approach to be followed in solving theoretical or real life problems. Robotic techniques may be too useful in order to understand abstract concepts (Wing, 2006). For example, while assembling parts of the Robotics kits, children begin to explore the Math concept of the ratio. They learn about electrical engines, sensors or about various mechanical parts, being able to create complex toys moving and reacting according to a certain design (Bers, 2008). Recently, Benitti (2012) reviewed the scientific literature related to the use of Robotics in schools, suggesting that Educational Robotics may be an important factor in improving Teaching process.

During two years (4th and 5th grades), two children classes from a public school in Bucharest (Gymnazial School 195) followed courses of Robotics and Applied Mathematics in Movement Transmission (AMMT). The two courses have been presented in addition to the normal curriculum, both groups consisting of 36 children. Robotics course used the Lego Mindstorm EV3 kit (http://mindstormes.lego.com/). A major goal of the above extracurricular activity was the development of the group relations and the increase of self-confidence of the small children. This could be put into practice by organizing discussions between children, brainstorming sessions to discuss the proposed solution and adopt the most appropriate one. The teachers also encouraged the originals ideas proposed by children. This paper presents quantitative and qualitative results regarding school performances evaluation for the two classes. Both the qualitative and quantitative analyses performed suggest better scholar performances of the children following Robotics courses, in respect to the ones following AMMT ones and a greater attraction of the children in Robotics.

The main objective of the research is to investigate the process by which students build their skills to solve mathematical problems and create skills to seek and find solutions based on technological situations encountered during the course. The research aims at the effects of learning mathematics based on the construction and programming of robots as a pedagogical means in guiding students during two years of school (4th grade and 5 th grade). These effects are analyzed both in terms of school performance, but also in terms of personal development and relationships with others in groups, where common topics are discussed and resolved. All these effects come as a result of the development of weekly courses and robotics projects such as LEGO Mindstorm EV 3 and in the accumulation of knowledge with the processes and methods of solving mathematical problems. 


\section{Theoretical considerations}

The term of Educational Robotics (ER) describes the use of Robotics as a method to learn and teach. It was introduced approximately 20 years ago. The first main direction of ER focused on teaching and learning higher technology. The second direction is related to the design of new projects, children becoming familiar to more and more difficult concepts by building the small robots. While the sophisticated robotics used in the great research laboratory is very expensive, the low cost of an ER kit makes the basic elements of such a technology affordable in an elementary school class too. Actually, a common robotic kit offers a programmable system, routinely based on graphic elements. It is enough simple to be used by the small children but also enough elaborated to allow users to design complex activities of the robots (Hendler, 2000)

A typical ER kit contains a smart brick, acting as the robot brain, various sensors (e.g. the ones for light, colour, distance) and various other elements to be attached to the main module. A popular ER kit is Mindstorm, designed by LEGO. At the first LEGO competition in 2009, around 14725 pieces were used (FIRST LEGO LEAGUE, 2011).

Most of the robotic kits provide a specific software, designed to guide the children in making the first steps toward computer programming by using advanced languages (e.g. JAVA, NQC, ROBOTC, LABWIEW). By increasing the programming level, Educational Robotics may be used both by the students in the elementary schools and in high schools too.

ER helps children learn Maths easier, scientific concepts and help them to develop technological abilities (Barker et al., 2012). There are various studies indicated such a kind of education provides opportunities of better understanding in fields like Physics, Biology, Geography or Maths. It is also useful in developing collaboration, making decisions and in solving various other problems.

Robotics inspires students to become interested in STEM field, meaning Science, Technology, Engineering and Mathematics. Barker et al. (2012) present some examples in which students who had difficulties in high school in mathematics then show interest in robotics courses and even success in this area. Working with robots inspires children to write extensively about their robotic projects, to do calculations or to be willing to learn mathematical concepts to help them make interesting programming and build problems. Barker et al. (2012) note the following:

In robotics courses, students are attracted to address the issues that have been presented to them or to their own design. The learning environment in which the student is engaged motivates him to acquire skills and acquire the knowledge necessary to achieve their goals. Students are attracted and involved in the challenges of building and programming to make real what they imagined ( pp.6).

Educational Robotics helps children interested in the STEM domain (Science, Technology, Engineering and Math), helping them to understand the basic elements of the so-called "computerized thinking" (Wing, 2006), a process involving (Barr et al., 2011): 
- Formulating problems in a way that enables us to use a computer and other tools to help solve them;

- Logically organizing and analyzing data;

- Representing data through abstractions, such as models and simulations;

- Automating solutions through algorithmic thinking (a series of ordered steps);

- Identifying, analyzing, and implementing possible solutions with the goal of achieving the most efficient and effective combination of steps and resources;

- Generalizing and transferring this problem-solving process to a wide variety of problems (pp.21).

Skills such as critical thinking and problem solving, communication, collaboration and creativity are the ones needed in the 21st century, according to the American Teachers Association (2010). Barr et al.(2011) observed that:

The long-term goal is to recommend ways that all students have the opportunity to learn these skills and to ensure that they can be transferred to different problems and used in different contexts (pp.23).

\section{Methodology}

The research adopted a complex methodology, both qualitative and quantitative ones, in order to show various aspects of the teaching/learning process and to outline possible correlations betwen them. This study follows to evaluate the improvement of the Maths performances of the children, both classes following the same teaching material for the lessons. The same Math curriculum has been used and, during the 5th grade, the Maths teacher was the same. The Maths tests used in evaluation was the same too, each test having a maximum score of 100 , which has been finally divided by 10 . There have been 36 children in each class and no social problems have been noted during the two years of the research. At the beginning of the classes, the children were given the Raven intelligence test. It was chosen the non-colored version of the test, consisting of 5 series (A, B,C,D,E) of 12 each items. The test is built to cover various levels of mental abilities and can be applied to all ages. Before the beginning of the classes, it was obtained the official approval from the school management in order to carry out these options, in optimal conditions. Also, it was obtained the approval from the parents to accept their children's participation in both courses and interviews. The confidentiality of the satisfaction questionnaires was ensured by handing them over anonymously. In the Gymnasia School Number 195, Bucharest, in the school year 2017-2018, there were eight $4^{\text {th }}$ grade classes. Among them, two were randomly selected, for participation in the two courses, subsequently obtaining the consent of teachers of both classes.

\section{Significant courses details}

Some significant details about the conduct of the two courses are:

- The children had the same textbooks and study materials in the Mathematics discipline. 
- Both classes followed the same program and planning in Mathematics.

- In the 2017/2018 school year, in the 4th grade, the children did Mathematics with the primary school teacher, which was different.

- In the school year 2018/2019, in the 5th grade, the children had the same teacher in Mathematics.

- The Mathematics tests that were applied to the students, 3 per year (at the beginning of the school year, in the middle and at the end of the school year), both for the 4 th and for the 5 th grade were the same for both participating classes to courses.

- All 12 tests were evaluated by scores, the maximum score being 100 points. The capitalization of the score in a school evaluation grade was done by dividing it by 10.

- Each class had in the school years 2017/2018 and 2018/2019 the same number of 36 children.

- The general climate of the classes was very good: there were no significant problems in learning in any of the subjects, of relationships between colleagues, between students - teachers and there were no social problems.

The programs after which the courses took place in both years of study can be seen in the Appendix 1, 2, 3, and 4.

\section{Results}

An important aspect in the analysis of qualitative and quantitative data is that students started at approximately the same level, as school and intellectual training. This fact is also found in the correlation of the scores of the intelligence tests given to the two groups at the beginning of the courses. The averages of the results of the intelligence tests are $(91+/-5) \%$ for the Robotics group and $(90+/-5) \%$ for the group that participated in the AMMT course. The difference between the two averages is not statistically significant at the level of confidence of 0.05 according to the t-test.

\section{The qualitative Analysis}

It was focused on several factors involved in the learning process, especially on the emotions and on the actions of the children related to the two courses. The direct observation followed the personal involvement of the children in solving the asked tasks (Barker, 2012). The direct observation and the qualitative interpretation have been done for two school years. Children noted their impressions about the courses in a class journal. They noted about the received information and about the most attractive aspects of their activity. They also completed individual files containing their opinions by the end of each session. Their opinions have been analyzed continuously, but also at the end of each school year. Finally, children completed questionnaires regarding their satisfaction in respect to the two courses. There have been short interviews too regarding their final 
opinions about the novelty of the information received, their pleasure and the enthusiasm achieved, the potential help in understanding the Maths notions. They have been questioned too in respect to the possible improvement of the group relations with their colleagues considering several tasks asked for such a children collaboration. Other questions were related to several aspects of the teaching activity and about the children intention to attend similar courses in future. Some parents also accepted to present their perception about the effects observed in children's behavior as a possible result of following the two facultative courses. So, the qualitative research consisted from the direct observation, from the files analysis and from the interview.

\section{The Direct Observation}

It outlined an increased enthusiasm of the children following the Robotics course when compared to the ones following the AMMT course. A possible explanation could be that the second group of children considered AMMT some sort of extension for the Maths regular hours, while the first group regarded Robotics as a complete new course. Another explanation could be the real life activities involved by the Robotics and the more theoretical characteristics of the AMMT lessons.

The behavior of the children illustrated clearly their preferences. The participants in Robotics systematically arrived earlier before the course hours in order to change impressions about the lessons. Such aspects have not been observed to the children attending the AMMT lessons. By the end of the Robotics hours, systematically, children from the first group used to remain further in the class, discussing the new information received and changing their impressions about the course. Similar discussions have been observed in the pause interval between the two course hours. Only a small number of children attending AMMT courses displayed a similar behavior, the pause interval being mostly treated as a routine one.

Absenteeism at the Robotics class hours was reduced when compared to the AMMT ones. Some of the children attending the Robotics course decided to buy LEGO MINDSTORMS EV3 kit from their own financial resources. They also asked the Robotics teacher to allow friends, brothers or sisters from other classes to be accepted at the lessons, which has not been observed in relation to the AMMT course. It shows the Robotics course has been highly appreciated among children.

\section{The Analysis of the Class Journals}

Two kinds of journals have been used, an individual one and a class one. Children have been asked to complete the individual journals by the end of each lesson, but writing in the class journal was not mandatory. The examination of the journals led to the following conclusions:

- the number of children writing in the class journal is greater for the Robotics class when compared to the AMMT class, indicating the Robotics course is more attractive;

- children attending the Robotic courses did explicit observations regarding the 
design, the quality of various sensors and robots programming in order to fulfill certain tasks;

- just a few observations have been noted by the children attending the AMMT course;

- the attraction of the children to the small robots has been observed by the drawings accompanying the notes in both journals. Such drawings represented an initiative of the children, being not asked by the Robotics teacher. Very few similar drawings accompanied the notes in the AMMT journals.

A direct conclusion is that the children of both classes showed an increased interest in relation to the real life aspects of the two courses when compared to the theoretical ones.

\section{Interviews Results}

A direct conclusion resulting from the discussions was that the Robotics course is more suitable for the children with the age in the range 10 to 12 years, when compared to the more theoretical ATTM course. Yet, both groups of children appreciated obtaining supplemental information on the fields of Physics, Logic and general knowledge.

For the Robotic course, the parents' interviews led to the following conclusions:

- a high level of consideration regarding the course and the increased interest of the children, mainly indicated by their intention to follow Robotics too during the next school year;

- many parents wanted to financially support such courses;

- some parents acquired individually the LEGO MINDSTORM $3 \mathrm{kit}$, following the children demand;

- parents indicated the children used to tell at home about the new activities performed at the Robotics course more frequently when compared to other courses.

Interviews of the children attending the Robotics course indicated the following:

- efficiency of the team working and of the inter-collegial relations as a direct result of the tasks asked to be solved during the course hours;

- high appreciation of the free discussions and the permanent exchange of the ideas regarding the course tasks;

- freedom in promoting some ideas, irrespective some opinions did not finally acquire a majority support;

- appreciation of the criticism and of the confrontation of ideas during solving the course tasks;

- appreciation about the final solutions resulted as a general effort of the whole group.

\section{The Quantitative Analysis}

The input data used here have been the individual scores at Maths obtained at the beginning, middle and the end of the two school years by each of the 36 children of the two classes. The scores have been statistically processed, the following parameters being 
evaluated for both classes : the arithmetic means, their errors (as an indicator of variance), the time evolution during the two school years of the children with the two lowest scores and the number of children obtaining the highest score. The means have been compared by using the Student's t-test (Barnes, 2008).

In the beginning, the arithmetic means and their errors (95\% confidence interval) have been evaluated for the 12 samples (Maths scores for the IV-th class and for the V-th class, at the beginning, at the middle, and at the end of each school year). The results are displayed in Figure 1 for each of the six moments in time.

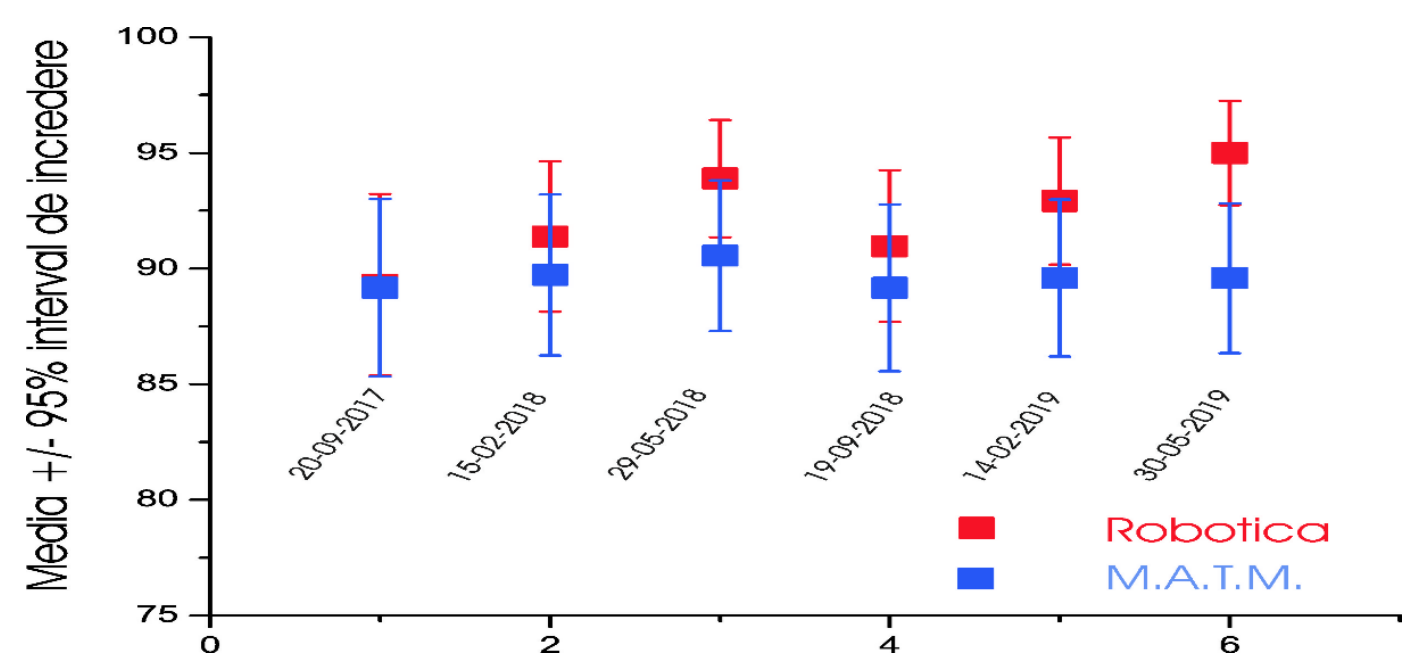

Figure 1

Chronological representation of the Maths means of the classes attending the Robotics course (red) and MATM (blue), with their bar errors (95\% confidence interval)

Figure 1 shows that for the initial moment (the beginning of IVth school year, just before the start of the Robotics and AMMT courses), the Math's evaluations show a quite similar level for the two classes, the arithmetic means of the two groups of children being very close to each other. So, the initial mean of the IVth class following to attend Robotics was 89.31 ( \pm 3.92 , 95\% confidence interval), while the corresponding values for the class following to attend the AMMT was 89.17 ( \pm 3.85 ). Figure 1 also indicates that the difference between the two mean values increased during both school years. The mean value for the Robotics class increased too in both years, suggesting a constant development of the school performance. Such process is hard to see in the first year for the AMMT class and is definitely not observed for the second year. It is most likely the result of a lack of interest for such a course.

Despite the two classes (Robotics and AMMT) started from the same quantitative level of Math performance, the differences between their average Math's scores is finally obvious. So, the Robotics class has a mean value of $95( \pm 2.25)$, while the AMMT has 89.58 $( \pm 3.24)$. With a probability greater than $95 \%$, Student's t-test shows that the first mean is significantly greater than the second mean.

Both in the IV-th and V-th classes, the variance of the Math scores illustrated by 
the $95 \%$ confidence interval errors decreased constantly, the values being presented in Table 1.

Table 1 Errors of the arithmetic means for the Math scores

\begin{tabular}{lllllll}
\hline $\begin{array}{l}\text { Course } \\
\text { Year }\end{array}$ & IV-th & IV-th & IV-th & V-th & V-th & V-th \\
Start & Middle & End & Start & Middle & End \\
\hline Robotics & 3.92 & 3.25 & 2.53 & 3.85 & 3.48 & 3.25 \\
\hline AMMT & 3.28 & 2.75 & 2.25 & 3.61 & 3.39 & 3.24 \\
\hline
\end{tabular}

Table 1 suggests a homogenization of Math performance for both classes during each year, more obvious for the Robotic class.

For each class, the evolution of the two children having the lowest Math scores is presented in Table 2.

Table 2: Lowest Math scores during the two years

\begin{tabular}{lllllll}
\hline $\begin{array}{l}\text { Course } \\
\text { Year }\end{array}$ & IV-th & IV-th & IV-th & V-th & V-th & V-th \\
Start & Middle & End & Start & Middle & End \\
\hline Robotics & $60 / 65$ & $70 / 75$ & $80 / 80$ & $70 / 70$ & $80 / 75$ & $80 / 85$ \\
\hline AMMT & $65 / 65$ & $75 / 65$ & $75 / 70$ & $70 / 70$ & $75 / 65$ & $75 / 70$ \\
\hline
\end{tabular}

Table 2 suggests that the two children having the lowest Math results from the Robotics class constantly improved their performances during both years. Their scores by the end of the Vth year are 20 points greater than the initial values. Such an evolution is less obvious for the AMMT class.

The evolution of the number of children obtaining the maximum number of points at the Maths tests is presented in Table 3.

Table 3: The number of maximum Math's scores during the two years

\begin{tabular}{lllllll}
\hline $\begin{array}{l}\text { Course } \\
\text { Year }\end{array}$ & IV-th & IV-th & IV-th & V-th & V-th & V-th \\
Robotics & 15 & Middle & End & Start & Middle & End \\
\hline AMMT & 15 & 13 & 18 & 14 & 17 & 19 \\
\hline
\end{tabular}

For the Robotics class, Table 3 suggests the number of children with highest performances at Math has a small positive jump between the middle and the final time period, but constantly increases during the second year. Regarding the AMMT class, the similar number constantly decreased during both study years, a possible explanation being the lack of interest associated with a more theoretical course.

\section{Limitations of the research}

The main limitations of the research are the following ones: 
- the relative small number of children / classes used, making questionable the generalizations of the results. This aspect is correlated to the financial costs involved by the Robotics course (the cost of the Robotic kits, of the teacher preliminary training and for the teacher's salary).

- a possible overload of the curriculum, considering that both Robotics and AMMT courses are extracurricular ones.

- the relative high financial cost of organizing a robotics course in a public scool, in both terms of purchasing robotic kits, but also in terms of financial remuneration for specialized teachers.

\section{Conclusions}

The result of the Statistical Analysis suggests an increase of the Math's performances for the children following the Robotics course when compared to the ones following the AMMT one. The performance is illustrated by a constant increase of the average score of the class, by a statistical homogenization of the group of children, by better results of the children with the lowest scores and by a greater number of maximum scores obtained.

So, Educational Robotics might be a non-traditional manner to increase the children's interest in learning. Working in a group, solving the practice problems together, children develop self-confidence, creativity and increased enthusiasm. In such a way, Robotics may become a strong educational way to prepare children for a computerized thinking asked by the modern society (Duckworth, 2005).

The results of this research suggest that Robotics should be introduced as extra-curricular activity in several pilot schools in the country and can support the adequacy of the education system to the needs of children and society. Yet, these needs must be balanced. Quality education is achieved with effort from the management of schools, teachers and with the help of supporters, parents, professional associations and Non-Governmental Organizations. Additional investments are needed in order these courses can be carried out in the field of education. So, in a relatively short time, this project will gain confidence, and Robotics will be introduced as an option school course, the main benefit being to cover a wide range of children from all segments of society.

\section{References}

Barr, D., Harrison, J., \& Conery, L. (2011). Computational Thinking : A digital age skill for everyone. Learning and Leading with Techonolgy, 38, 20-23

Barker, B., Nugent, G., Grandgenett, N., \& Adamchuk, V. (2012). Robots in K-12 Education. A new technology for learning, IGI Global, USA

Barnes, B. (2008). Mathematical modelling with cases studies, CRC Press, New York

Benitti, F. B. V. (2012). Exploring the educational potential of robotics in schools: A systematic review. Computers \& Education, 58(3), 978-988

Bers, M.U. (2008). Blocks to robots: Learning with technology in the early childhood classroom. New York: Teachers College Press.

Duckworth, E. (2005), Critical Exploration in the classroom, New Educator https://www.tandfonline.com/doi/abs/10.1080/15476880500276728 
Hendler, J. (2000), Robots for kids : Exploring new techonologies for learning (pp. 2-7), San Diego, Academic Press

Martin, F., Mikhak, B., Resnick, M., Silverman, B., \& Berg, R. (2000). To mindstorms and beyond: Evolution of a construction kit for magical machines. Exploring new technolgies for learning (pp. 9-33), San Francisco: Morgan Kaufmann.

Rogers, C. \& Portsmore, M. (2004). Bringing engineering to elementary school, Journal STEM Education: Innovations and Research, 5(3-4), 17-28.

Rogers, C.B., Wendell, K. \& Foster, J. (2010). The Academic Bookshelf: A Review of the NAE Report, Engineering in K12 education, Journal of Engineering Education, 99(2), 179-181.

Rusk, N., Resnick, M., Berg, R., \& Pezalla-Granlund M. (2008). New pathways into robotics: strategies for broadening participation. Journal of Science Education and Technology, 17, 59-69.

Waterhouse, S. (2005). The power of e-learning: The essential guide for teaching in the digital age, Boston, MA: Pearson.

Wing, J.M. (2006). Computational thinking. Communications of the ACM, 49 (3), 33-35. http://www.cs.cmu.edu/afs/cs/usr/wing/www/publications/Wing06.pdf

\section{APPENDIX 1}

\section{Robotics Year I (2017-2018)}

The 3rd generation LEGO Mindstorm kit allows in an interactive / intuitive way the assimilation of the basic notions of visual programming of computers. It has an immediate effect and applicability in the movement and interaction with the environment of an assembly that fully fulfills the notion of a robot. In the last 10 years since the phenomenon spread all over the world, it has been observed in carefully monitored studies, that during the course sessions, groups of pupils / students regardless of age target develop cognitive, social and communication skills. They find solutions much faster through abstract thinking, due to the fact that the theoretical notions learned are immediately applied. The course aims to teach the target group notions of visual programming of computers, elements of motion mechanics, understanding the notion of data acquisition, application of mathematical models in describing and replicating phenomena reproduced by a machine during twenty sessions of $2 \mathrm{~h}$ each.

1. Introductory Course. Brief history of the tools used in human history up to independent programmable machines; description of the Mindstorm kit; installation and configuration of specific software on the personal computer; first program: touch sensor reading.

2. Specific movement elements, gears; assembling the first robot. Its connection to the servomotors. Program: movement of the robot in a circle.

3. Introductory programming course. Transposing a problem into a logical scheme; identifying the code blocks needed to replicate the schema in a program.

4. Insert the color sensor. Presentation of the 3 modes of operation, assimilation of the notion of reflected light intensity. Understanding how computing machines "understand" the environment. Basic notions of data acquisition; mathematical problematic situations. 5. Assimilation of the notion of variables and their allocation in available memory locations. Elementary notions of initialization and increment / decrement with constant values + mathematical problematic situations. 
6. Color detection. Development of a program through which the robot previously built as a base, recognizes the elementary colors by moving over a colored surface. Reporting them on the smart-brick display in a table + mathematical problem situations.

7. The program "follow" the line. Purpose: the continuous movement of a robot on the demarcation line between two contrasting colors. Notions: logical scheme, transposition into blocks of code, the notion of infinite loop; mathematical problematic situations.

8. The experimental way of adjusting the parameters applied on the previous mathematical model. Introducing the proximity sensor. Data acquisition of the sensor when converting the distance into numerical values + mathematical problematic situations.

9. Deepening the notions of calibration / parameterization based on experiment. The difference between the theoretical application of the mathematical model on the robotic machine and the actual operation + mathematical problematic situations.

10. Color sensor calibration program. Its usefulness in changing the operating conditions and the effect of ambient light on the "follow the line" program + mathematical problematic situations.

11. Inserting the proximity sensor into the previously assembled machine. Putting the robot in the decision-making situation to avoid random obstacles + mathematical problematic situations.

12. Case study: solving a maze by using the proximity sensor. Use of subroutines. The logical scheme of the program.

13. Effective programming of the previous model. Empirical adjustment of parameters + mathematical problematic situations.

14. The mechanics of a robot "hand" that grabs and releases objects. Presentation of the mechanisms: "worm wheel worm" and "eccentric" + mathematical problematic situations.

15. Deepening the learned mechanisms by applying them in a "Spirograph" model that draws in a repetitive way. Tracking the effect of various parameters in the mechanics of motion + mathematical problematic situations.

16. Programming the subroutine specific to the movement of the robotic arm. Empirical measurement of the rotations necessary for the micro motor to achieve the "grab" and "release" effect + mathematical problematic situations.

17. Presentation of the final theme. Replication of the "amazon warehouse" example of arranging goods in a fully robotic warehouse.

18. Presentation of the plan / route. The objective pursued is: moving from a starting point of the robot on a predetermined route, detecting the "goods" stop / grab / return on the same route, release on the starting point + mathematical problematic situations. 19. Adjusting the "primitive" subroutines previously developed: "follow the line" and "grab / lift" for simultaneous operation. The need to introduce "flag" variables to achieve the objective + problem situations. 


\section{APPENDIX 2}

\section{Robotics Year II (2018-2019)}

1. Introductory course. Review of the main knowledge learned at the Robotics courses in the previous school year and bringing back into discussion the main team projects carried out.

2. Presentation and discussion of interviews with students participating in the international Robotics Olympics and competitions around the world. Observation of certain projects, ideas and designs of participating and winning robots in different years of international competitions and Olympics.

3. Using the proximity sensor in writing programs, for the robot to follow the path of a maze, being at certain distances from the walls: $10 \mathrm{~cm}, 15 \mathrm{~cm}, 20 \mathrm{~cm}$. Finding solutions for the situation in which the robot wanders far forward or backward. Challenge: finding a way to improve the program so that the wall path is faster + solving possible math problems that require solving the topic.

4. Creating custom blocks in EV3 (My Block) software. The usefulness and construction of a custom block on groups with inputs and outputs. Update the EV3 application for such exercises. Challenge: moving the robot according to certain distances given in certain times.

5. Scroll through a color line in the custom block with several entries. Writing the program to trace a color line, which stops after a certain number of degrees. Changing the input when you want to follow a route over different distances. Adjusting the speed of the robot to a certain direction.

6. Using the infrared sensor. Test Infrared sensor for measuring distances. Applications on mathematical exercises and problems. Limitations of the infrared sensor.

7. Experiment with the different modes of movement of the robot. Mathematical calculations to avoid obstacles in the way of the robot.

8. Remediation techniques. Using useful strategy (debugging) in the situation in which something goes wrong in the program. Finding out the values different sensors sees.

9. Improving the reliability of the program. Finding possible solutions to increase the reliability of the program. Characteristics of the robots participating in competitions.

10. Calibration of color sensors. Using the EV3 color sensor in light sensor mode (reflected light mode) and its calibration. Writing a custom program for different color sensors (equations and mathematical situations).

11. Types of variables. Learning, reading and writing: data, sensors color, display blocks, and color blocks.

12. Proportional control. Learning to apply proportional control to different sensors. Solving math blockages and calibrating the sensor color.

13. Deepening proportional control. Exercises and mathematical situations.

14. Introduction of the sound sensor in the proportional control. 
15.Bluetooth functionality. Learning about radio frequencies as well as ways communication between devices. Learning to receive and send messages between EV3 Mindstorm bricks.

16. Challenges: different exercises and problems given to students to be solved and discussed within the group.

17. Deepening challenges: different exercises and problems given to students to be resolved and discussed within the group.

18. Choosing and discussing the preparation of the final project.

19. Discussing and resolving any difficulties encountered during the course implementation of the project.

20. Presentation and discussion of projects.

\section{APPENDIX 3}

\section{Applied Mathematics in Motion Transmission Year I (2017-2018)}

1. Introductory course. Review of the mathematical knowledge.

2. Problems finding the unknown number.

3. Problems that are solved by more than three known operations.

4. Graphic representation method.

5. Method of comparison / reduction per unit.

6. Discrete quantity method.

7. Reverse method.

8. Motion problems (distance / speed / time). Problematic situations.

9. Deepening problems. Calculation of distance / speed / time.

10. Problematic speed calculation situations.

11. Probability method.

12. Problem solving by combined methods.

13. Calculation of area / volume. Problematic situations.

14. Problems with fractional / decimal / percentage numbers.

15. About simple electrical circuits, for children to understand. Experiments.

16. About simple electrical circuits (deepening).

17. Problems of calculating time intervals.

18. Problem solving by combined methods (deepening).

19. Project "Mathematical surprises around us".

20. Review and evaluation of the M.A.T.M. 


\section{APPENDIX 4}

\section{Applied Mathematics in Motion Transmission Year II (2018-2019)}

1. Introductory course. Review of mathematical knowledge.

2. The power of numbers. Calculation exercises.

3. Complex power calculation problems.

4. Problems of removing integers from a fraction.

5. Estimation and approximation problems.

6. Logic problems and probabilities.

7. Combined methods for solving arithmetic problems.

8. Combined methods for solving arithmetic problems (consolidation).

9. Combined methods for solving arithmetic problems (deepening).

10. Simple motion transmission systems.

11. Simple motion transmission systems. Mathematical situations.

12. Problems of deepening the movement. Speed / distance calculation (consolidation).

13. Problems of deepening the movement. Speed / distance calculation (deepening).

14. Complex problems of calculating various time intervals (consolidation).

15. Complex problems of calculating various time intervals (deepening).

16. Problems in which the furniture has opposite meanings ("meeting problems").

17. Problems in which the furniture has the same direction of movement ("tracking problems").

18. Problems finding the area / volume in various geometric constructions.

19. Project "Mathematics is in EVERYTHING".

20. Review / evaluation of the M.A.T.M. 